Ann. Biol. anim. Bioch. Biophys., I974, 14 (2), 293-311.

\title{
ÉTUDE DE LA VARIATION DU NOMBRE ET DES DIMENSIONS DES FIBRES MUSCULAIRES CHEZ TROIS RACES DE LAPINS ET LEURS CROISEMENTS RÉCIPROQUES (')
}

\author{
J. NOUGUÈ, Anne-Marie ROUS, P. VIGNERON \\ avec la collaboration technique de Odette Moulierac et L. MARGER \\ Station de Physiologie animale, \\ Ecole nationale supérieure agronomique, I. N.R. A., \\ Place Viala, 34060 Montpellier Cedex
}

RÉSUMÉ

L'objet de l'étude est d'analyser la variation, chez 3 races de lapins (Commun gris, NéoZélandais, Blanc de Bouscat) et leurs croisements réciproques, de certaines caractéristiques histologiques des muscles soleus, accessorius latissimi dorsi, extensor carpi vadialis longus et stylo-hyoideus.

Les caractères étudiés sont le poids du muscle, le nombre total des fibres musculaires et leurs dimensions moyennes (aire de section transversale et longueur). Les deux premiers caractères sont mesurés sur les 4 muscles. Les 2 derniers sont mesurés seulement sur les muscles soleus et accessorius latissimi dorsi.

Une analyse de variance, sur les données obtenues, montre que pour les muscles, soleus, extensor carpi radialis longus et stylo-hyoideus, l'appartenance à une race ou un croisement donné a une influence sur le nombre total de fibres et le poids du muscle, et, pour le muscle accessorius latissimi dorsi, une influence sur les dimensions des fibres et le poids du muscle. Puis une analyse factorielle discriminante permet de séparer les races et les croisements, en explicitant les variables responsables de cette séparation.

Les résultats de cette analyse montrent que, pour le muscle soleus, c'est le caractère "nombre total de fibres " qui est différent entre les trois races. Il présente les valeurs les plus élevées chez les lapins Néo-Zélandais et les plus faibles chez les lapins Communs. Le muscle soleus des produits des croisements présente des caractères intermédiaires ou proches de ceux des races, dont ils sont issus.

Par contre, pour le muscle accessorius latissimi dorsi, ce sont les dimensions des fibres qui sont différentes entre les trois races et certains croisements, mais uniquement chez les lapins femelles. Le muscle des individus Néo-Zélandais se caractérise par des fibres de grande aire de section transversale et celui des individus Communs par un poids plus élevé en liaison avec des fibres de grande longueur. Les muscles des lapins hybrides ne présentent pas de caractères intermédiaires de ceux des parents, dont ils sont issus.

Enfin, les muscles extensor carpi vadialis longus et stylo-hyoideus des individus de la race Néo-Zélandaise sont différents de ceux des individus de la race Commune par un plus grand nombre de fibres, sauf en ce qui concerne le muscle extensor carpi vadialis longus des lapins femelles. Les

(1) Ce travail a été effectué avec le concours financier de la Délégation générale à la Recherche scientifique et technique (contrat $n^{\circ}$ ᄀI 73 I29). 
muscles des individus Bouscat présentent des caractères intermédiaires entre ceux des $N e ́ o-Z e ́ l a n-$ dais et des Communs. Il ressort donc de cette étude que les caractéristiques histologiques d'un muscle, en particulier le nombre des fibres, sont susceptibles de présenter des variations d'origine génétique, entre races, chez lc Lapin.

\section{INTRODUC'TION}

Les caractères structuraux les plus importants dans la détermination du poids d'un muscle sont le nombre et les dimensions des fibres musculaires, 1'ensemble de ces derniers éléments constituant, chez les petits mammifères, de l'ordre de $85 \mathrm{p}$. Ioo du poids du muscle. Or les résultats d’un travail précédent Nouguìs (I972), sur 1'étude de l'évolution du nombre des fibres pendant la croissance musculaire du Lapin commun, ont permis de constater que ce caractère est fixé précocément ; il est, de ce fait, peu soumis aux influences de l'environnement. Malgré cela, il présente une variabilité assez importante en relation avec des facteurs génétiques.

Par ailleurs, des résultats bibliographiques variés relatent l'influence de la race et des croisements entre races sur la variation des caractères nombre et diamètre des fibres musculaires. Certains auteurs n'ont étudié que le diamètre des fibres ; ainsi, Hammond et Appléton (I932) chez le Mouton, March et Marinesco (I934) chez le Porc ont observé que ce caractère était plus élevé chez les races améliorées que chez les races rustiques. JOUBERT ( 1956 ) a remarqué une différence significative du diamètre des fibres musculaires entre les races bovines Dairy Shorthorn et Friesian et leurs croisements avec les Angus et Hereford. Par contre, DoRozynska et I Iszka (I970) n'ont pas observé de différence significative du diamètre des fibres musculaires $\mathrm{du}$ longissimus dorsi et du rectus femoris chez des lapins de races différentes (Géant de Flandres et Chinchilla) et leurs croisements réciproques.

D'autres auteurs ont étudié les deux caractères nombre et diamètre des fibres ; ainsi, Mizuno et Hikami (I967) n'ont pas remarqué une différence du nombre des fibres du muscle pectoralis superficialis entre embryons de Poulet de diverses souches. SмIтн (r963) a fait la même observation pour le nombre des fibres du muscle sartorius de deux races de Poulet, mais cet auteur a constaté que la différence du poids des muscles entre les races, s'explique surtout par une différence du diamètre des fibres musculaires; les croisements réciproques présentent des valeurs intermédiaires de celles des parents. Chez les souris, LUFF et GoLDsPINK (I967), comparant les muscles de sujets appartenant à des souches naines et géantes, ont observé que les différences pondérales entre les muscles des deux souches proviennent davantage d'une différence de nombre que des dimensions des fibres. Enfin STAUN (I963) mesurant les caractères précités sur une section transversale du longissimus dorsi de porcs de diverses races, caractérisés par des rendements en viande inégaux, a constaté que ces caractères sont différents selon les races.

La plupart de ces travaux ne tiennent compte au maximum que de 3 caractères du muscle : son poids, le nombre et le diamètre des fibres. Certains sont imprécis, soit du fait du matériel animal utilisé, ce qui est le cas chez les gros mammifères domestiques, où la grande taille des muscles rend difficile la mesure d'un caractère tel que 
le nombre des fibres, soit du fait des techniques utilisées, la plupart des auteurs estimant le nombre des fibres sur une coupe transversale du muscle qui ne les contient pas toutes; des variations importantes de ce caractère en relation avec une diversité raciale de la forme du muscle peuvent passer inaperçues.

Enfin, aucun de ces travaux ne tient compte à la fois des 4 caractères suivants : poids du muscle, nombre, aire de section et longueur des fibres. Nous avons donc réalisé une étude de ces 4 caractères sur 3 races de lapins et leurs croisements réciproques.

\section{MATÉRIEL ET MÉTHODËS}

Les lapins mâles et femelles utilisés dans cette étude appartiennent aux races dites Commune grise, Néo-Zélandaise et Blanc de Bouscat et aux produits de leurs croisements réciproques. Les effectifs des divers groupes sont présentés dans le tableau I. Tous les croisements avec la race Blanc de Bouscat n'ont pu être étudiés, du fait de la disparition de reproducteurs ayant montré des difficultés d'adaptation à l'élevage sur grilles. Dans la nomemclature des produits de croisement, nous avons toujours présenté la race paternelle suivie de la race maternelle.

TABLEAU I

Plan de croisements et effectifs expérimentaux

\begin{tabular}{|c|c|c|c|}
\hline \multirow{2}{*}{ 우 } & \multicolumn{2}{|c|}{$\sigma^{*}$} & \multirow[b]{2}{*}{$\begin{array}{c}\text { Blanc de Bouscat } \\
\text { (3) }\end{array}$} \\
\hline & $\begin{array}{c}\text { Commun } \\
\text { (1) }\end{array}$ & $\begin{array}{c}\text { Néo-Zélandais } \\
\text { (2) }\end{array}$ & \\
\hline $\begin{array}{c}\text { Commun } \\
\text { (1) }\end{array}$ & $\begin{array}{ll}5 & \text { đ̊ } \\
5 & \text { ૧ }\end{array}$ & $\begin{array}{ll}5 & 0 \\
5 & 0\end{array}$ & \\
\hline $\begin{array}{c}\text { Néo-Zélandais } \\
\text { (2) }\end{array}$ & $\begin{array}{ll}5 & \text { む } \\
5 & 0\end{array}$ & $\begin{array}{ll}5 & 8 \\
5 & \text { q }\end{array}$ & $\begin{array}{ll}6 & 8 \\
5 & 9\end{array}$ \\
\hline Blanc de Bouscat & & 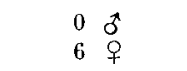 & $\begin{array}{ll}4 & \delta \\
7 & 9\end{array}$ \\
\hline
\end{tabular}

Les muscles soleus, accessorius latissimi dorsi, extensor carpi radialis longus et stylo-hyoideus ont été prélevés sur les animaux mâles et femelles sacrifiés à un poids vif de 2 ooo g. Ce poids est atteint par les individus des différents croisements et races vers l'âge de 70 jours, où le nombre de fibres des muscles est définitivement fixé (Nougù̀s, I972).

Les caractères poids du muscle et nombre total de fibres ont été mesurés sur les 4 muscles, et les caractères aire de section transversale et longueur des fibres ont été mesurés seulement sur le soleus et l'accessorius latissimi dorsi gauches, les muscles du côté droit étant utilisés pour la détermination du nombre des fibres. Une étude préalable a montré que le nombre de fibres et le poids de l'accessorius latissimi dorsi et du stylo-hyoideus ne sont pas significativement différents entre les muscles contralatéraux. On peut donc comparer des dimensions des fibres obtenues sur un muscle avec le nombre de fibres mesuré sur son symétrique.

Les mesures du nombre total des fibres, de leur aire de section transversale et de leur longueur moyennes ont été réalisées selon des techniques déjà décrites (Nouguks, I972 et I973).

Pour chaque muscle étudié, les analyses statistiques des données ont été réalisées sur un ordinateur IBM I I 30 (Laboratoire de Biométrie, I. N. R. A., Champenoux). Elles consistent en une 
analyse de variance, permettant de comparer les fluctuations des caractères observés autour de la moyenne générale et autour des moyennes des groupes correspondant aux divers croisements et races, et en une analyse factorielle discriminante permettant de séparer ces groupes en explicitant les variables responsables de cette séparation.

\section{RÉSULTATS}

L'analyse des données a été effectuée pour les lapins mâles et femelles groupés et considérés séparément. Les valeurs moyennes et les écarts-types des données sont présentés dans le tableau 2 pour les muscles soleus et accessorius latissimi dorsi et dans le tableau 3 pour les muscles extensor carpi radialis longus et stylo-hyoideus.

\section{I. - Analyse de variance}

Les résultats de l'analyse de variance sur les données obtenues pour les 4 muscles des lapins mâles et femelles groupés sont présentés dans le tableau 4. Ils permettent de dégager les observations suivantes :

Pour chacun des 4 muscles étudiés, les poids moyens des muscles de certains groupes sont significativement différents de la moyenne générale. Les muscles soleus et accessorius latissimi dorsi se comportent d'une manière dissemblable pour les caractères nombre de fibres et dimensions des fibres. En effet, pour l'accessorius latissimi dorsi le nombre moyen de fibres des muscles des divers groupes ne diffère pas significativement de la moyenne générale, alors que pour ce même muscle les dimensions moyennes des fibres de certains groupes sont significativement différentes de la moyenne générale. Des caractéristiques inverses sont observées pour le muscle soleus. Enfin, pour tous les muscles, à l'exception de l'accessorius latissimi dorsi, les valeurs moyennes du nombre total des fibres de certains groupes sont significativement différentes des moyennes générales.

En résumé, pour les muscles soleus, extensor carpi radialis longus et stylo-hyoideus, l'appartenance à une race ou un croisement donné, a une influence sur le nombre total de fibres et le poids du muscle et, pour le muscle accessorius latissimi dorsi, une influence sur les dimensions des fibres et le poids du muccle.

\section{2. - Analyse factorielle discriminante}

Nous avons pour les muscles soleus et accessorius latissimi dorsi des données correspondant aux 4 caractères : $x_{1}=$ poids du muscle, $x_{2}=$ nombre total des fibres, $x_{3}=$ aire moyenne de section transversale et $x_{4}=$ longueur moyenne des fibres, mesurés sur 7 groupes de lapins, correspondant aux 3 races et aux 4 croisements étudiés.

Les matrices de corrélations entre les caractères sont calculées intragroupes pour les individus mâles et femelles considérés séparément et ensemble.

Le tableau 5 présente les résultats relatifs aux lapins mâles ou femelles. On constate, pour le soleus, qu'il existe une corrélation positive significative entre le poids du muscle et le nombre des fibres, cette corrélation étant plus élevée chez les individus mâles, qui présentent, par ailleurs, une corrélation positive significative entre le poids 


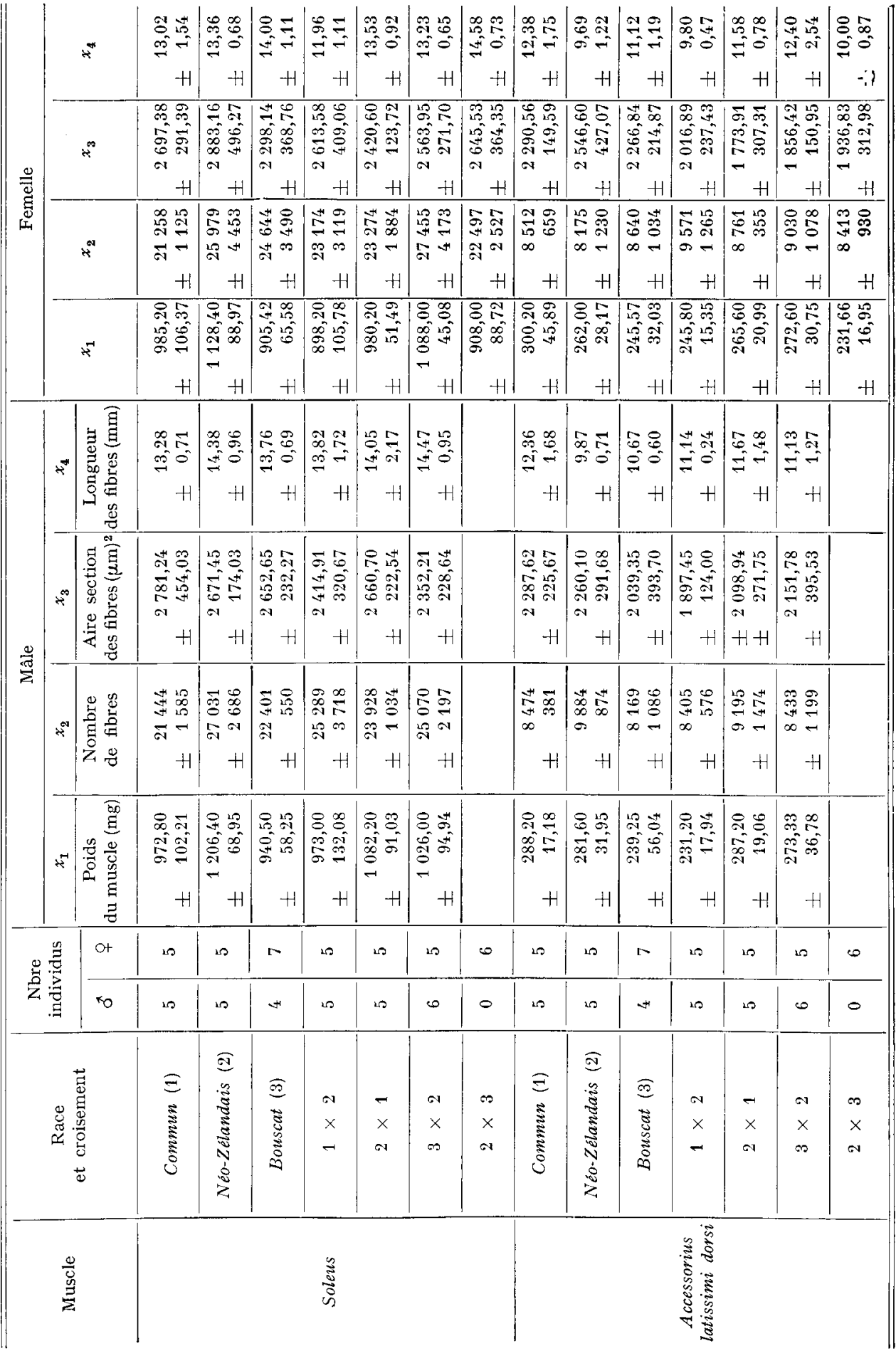




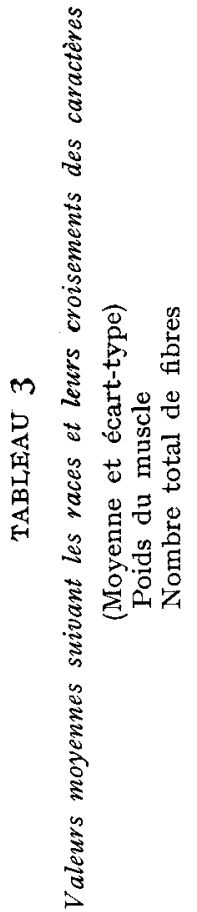

\begin{tabular}{|c|c|c|c|c|c|c|c|c|c|c|c|c|c|c|c|}
\hline \multirow{2}{*}{ 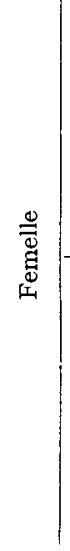 } & 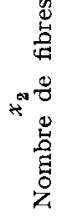 & 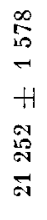 & \begin{tabular}{|c|}
$\infty$ \\
0 \\
0 \\
$\infty$ \\
$\infty$ \\
$H$ \\
$H$ \\
0 \\
$\infty$ \\
$\infty$ \\
$\infty$ \\
$\vec{\sigma}$
\end{tabular} & 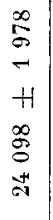 & 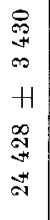 & 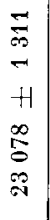 & $\begin{array}{l}2 \\
0 \\
0 \\
\infty \\
\infty \\
H \\
0 \\
0 \\
0 \\
0 \\
0 \\
0 \\
0\end{array}$ & 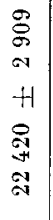 & 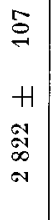 & 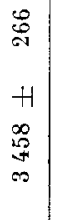 & 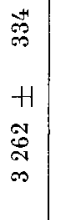 & $\begin{array}{l}\vec{\infty} \\
H \\
H \\
\vec{\infty} \\
\vec{\infty}\end{array}$ & 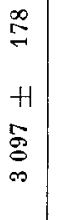 & 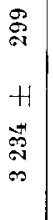 & 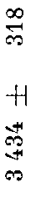 \\
\hline & 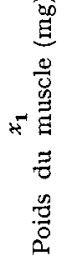 & 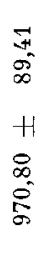 & \begin{tabular}{l}
\multirow{2}{*}{} \\
0 \\
0 \\
0 \\
$H$ \\
8 \\
0 \\
0 \\
0 \\
0
\end{tabular} & 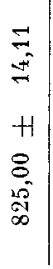 & 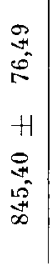 & 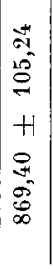 & 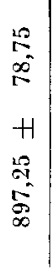 & $\begin{array}{l}0 \\
0 \\
\infty \\
\infty \\
0 \\
H \\
8 \\
0 \\
0 \\
0 \\
\infty\end{array}$ & 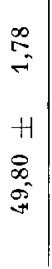 & $\mid \begin{array}{c}0 \\
\infty \\
\infty \\
H \\
H \\
8 \\
0 \\
0 \\
0\end{array}$ & $\begin{array}{l}\infty \\
\infty \\
0 \\
10 \\
H \\
H \\
11 \\
\infty \\
\infty \\
\infty \\
-1\end{array}$ & 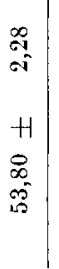 & 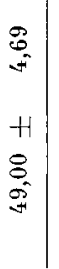 & $\begin{array}{l}0 \\
0 \\
0 \\
H \\
0 \\
0 \\
0 \\
0 \\
0\end{array}$ & $\begin{array}{l}\text { s } \\
\text { s. } \\
+1 \\
+ \\
0 \\
0 \\
0 \\
0\end{array}$ \\
\hline \multirow{2}{*}{ 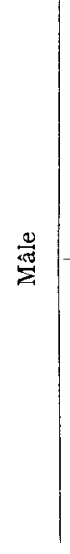 } & 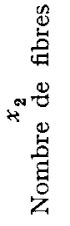 & 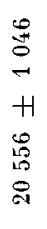 & 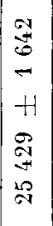 & 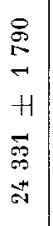 & 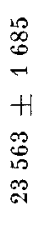 & 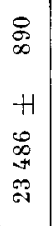 & 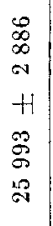 & & \begin{tabular}{l|}
$\stackrel{\infty}{N}$ \\
$H$ \\
+1 \\
$\stackrel{8}{\circ}$ \\
$\sim$
\end{tabular} & $\begin{array}{l}\infty \\
\vdots \\
+ \\
+ \\
8 \\
8 \\
\infty \\
\infty\end{array}$ & 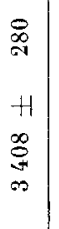 & 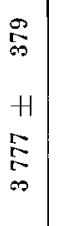 & 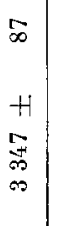 & 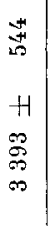 & \\
\hline & 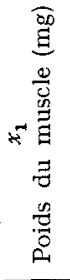 & 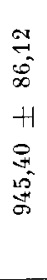 & 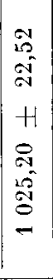 & 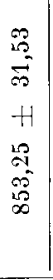 & 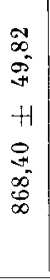 & 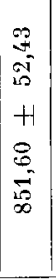 & 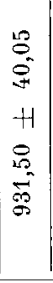 & & 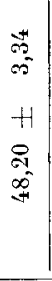 & $\begin{array}{l}0 \\
\infty \\
\infty \\
+1 \\
\infty \\
\infty \\
\infty \\
0 \\
0\end{array}$ & $\begin{array}{l}5 \\
\infty \\
+1 \\
1 \\
20 \\
\infty \\
\infty\end{array}$ & $\begin{array}{l}\infty \\
20 \\
-1 \\
+1 \\
\infty \\
-10\end{array}$ & $\begin{array}{l}5 \\
5 \\
H \\
8 \\
-5 \\
-5\end{array}$ & \begin{tabular}{l}
$\infty$ \\
0 \\
-1 \\
$H$ \\
0 \\
0 \\
0 \\
\multirow{\sigma}{*}{}
\end{tabular} & \\
\hline \multirow{2}{*}{ 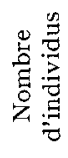 } & ot & סמ & is & $n$ & is & 10 & in & 0 & is & 10 & $r$ & 20 & is & is & 0 \\
\hline & to & is & 10 & $\omega$ & $\infty$ & is & 0 & 0 & is & is & + & 10 & 10 & 0 & $\theta$ \\
\hline \multicolumn{2}{|c|}{ 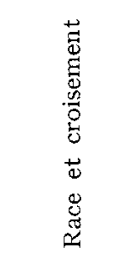 } & $\begin{array}{l}E \\
\bar{E} \\
\text { है } \\
\text { है }\end{array}$ & 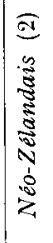 & 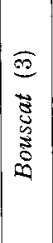 & $\mid \begin{array}{l}a \\
x \\
-\end{array}$ & $\left\{\begin{array}{c}-1 \\
x \\
a\end{array} \mid\right.$ & $\mid \begin{array}{l}\infty \\
x \\
\infty\end{array}$ & $\begin{array}{l}\infty \\
x \\
\infty\end{array}$ & 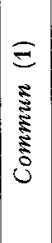 & 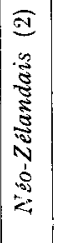 & 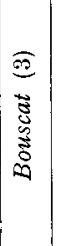 & $\begin{array}{l}a \\
x \\
-r\end{array}$ & $\begin{array}{l}\vec{x} \\
x \\
\text { a }\end{array}$ & $\begin{array}{l}\infty \\
x \\
\infty\end{array}$ & $\begin{array}{l}\infty \\
x \\
a\end{array}$ \\
\hline & 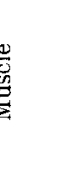 & \multicolumn{7}{|c|}{ 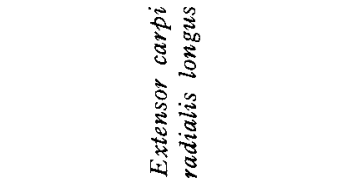 } & \multicolumn{7}{|c|}{ 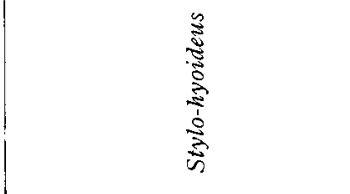 } \\
\hline
\end{tabular}




\section{TABLEAU 4}

Résultats de l'analyse de variance (valeurs de F) pour les données relatives à l'ensemble des lapins mâles et femelles

\begin{tabular}{|c|c|c|c|c|}
\hline \multirow[b]{2}{*}{ Muscles } & \multicolumn{4}{|c|}{ Caractères } \\
\hline & Poids $\begin{array}{c}x_{1} \\
(\mathrm{mg})\end{array}$ & $\begin{array}{l}x_{2} \\
\text { Nombre total } \\
\text { de fibres }\end{array}$ & $\begin{array}{c}x_{3}(\mu \mathrm{m})^{2} \\
\text { Aire moyenne } \\
\text { de section des fibres }\end{array}$ & $\begin{array}{c}x_{4} \\
\text { Longueur moyenne } \\
\text { des fibres }(\mathrm{mm})\end{array}$ \\
\hline Soleus & $10,11 * *$ & $4,21 * *$ & 1,80 & 1,73 \\
\hline $\begin{array}{c}\text { Accessorius } \\
\text { latissimi dorsi }\end{array}$ & $5,38^{* *}$ & 0,59 & $3,37 * *$ & $5,12 * *$ \\
\hline $\begin{array}{l}\text { Extensor carpi } \\
\text { radialis longus }\end{array}$ & $7,69 * *$ & $3,99^{* *}$ & & \\
\hline Stylo-hyoideus & $3,86^{* *}$ & $4,79 * *$ & & \\
\hline
\end{tabular}

Seuils de signification: ** 0,01 .

\section{TABLEAU 5}

Matrices des corrélations intra pour les muscles soleus et accessorius latissimi dorsi des individus mâles et femelles

(* significatifs $\mathrm{P}<0,05)$

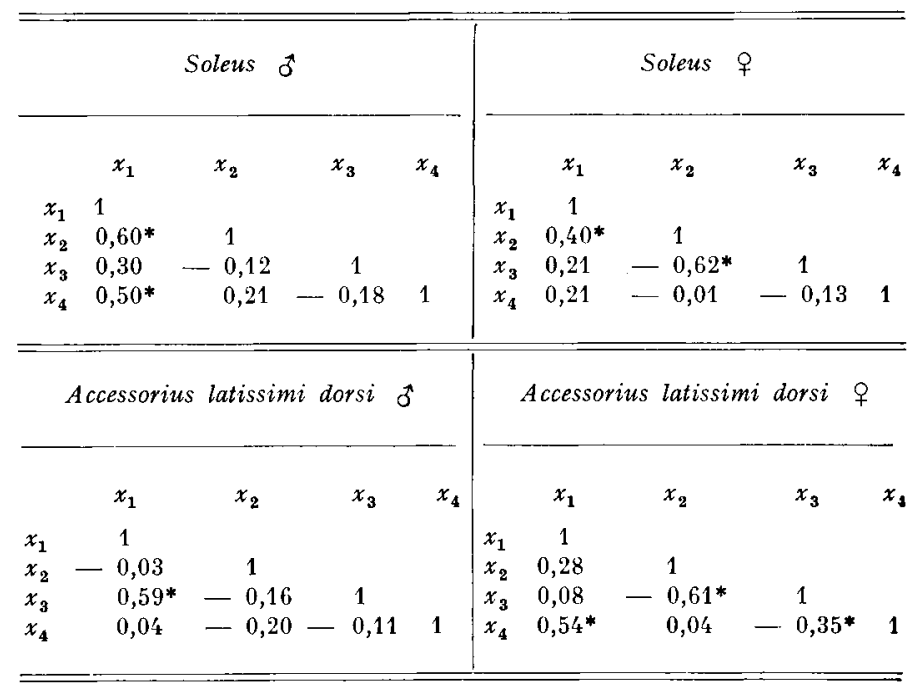




\section{TABLEAU 6}

Valeurs de $F$ correspondant aux distances généralisées de Mahalanobis calculées, entre les valeurs moyennes des individus des différents groupes, sur les caractères des muscles soleus (au-dessous de la diagonale) et accessorius latissimi dorsi (au-dessus de la diagonale).

\begin{tabular}{|c|c|c|c|c|c|c|c|}
\hline & 1 & 2 & 3 & $1 \times 2$ & $2 \times 1$ & $2 \times 3$ & $3 \times 2$ \\
\hline 1 & 0 & $\begin{array}{c}\text { NS } \\
4,72^{*} \\
9,49^{* *}\end{array}$ & $\begin{array}{c}\mathrm{NS} \\
4,30^{*} \\
8,28^{* *}\end{array}$ & $\begin{array}{c}\mathrm{NS} \\
5,96^{*} \\
6,00^{* *}\end{array}$ & $\begin{array}{l}\text { NS } \\
-\end{array}$ & $\frac{\text { NS }}{-}$ & $\begin{array}{l}\text { NS } \\
-\end{array}$ \\
\hline 2 & $\begin{array}{c}5,16^{*} \\
\text { NS } \\
8,31^{* *}\end{array}$ & 0 & $\begin{array}{c}\text { NS } \\
4,09^{*} \\
6,70^{* *}\end{array}$ & $\begin{array}{l}\text { NS } \\
-\end{array}$ & $\begin{array}{c}\text { NS } \\
9,23^{* *} \\
\text { NS }\end{array}$ & NS & $\begin{array}{c}\text { NS } \\
10,47^{* *} \\
\text { NS }\end{array}$ \\
\hline 3 & $\begin{array}{l}\text { NS } \\
- \\
-\end{array}$ & $\begin{array}{c}\text { NS } \\
4,63^{*} \\
10,12^{* *}\end{array}$ & 0 & $\begin{array}{l}\text { NS } \\
-\end{array}$ & $\begin{array}{l}\text { NS } \\
-\end{array}$ & $\frac{\mathrm{NS}}{-}$ & $\begin{array}{l}\text { NS } \\
-\end{array}$ \\
\hline $1 \times 2$ & $\frac{\text { NS }}{-}$ & $\begin{array}{c}\text { NS } \\
8,85^{* *} \\
\text { NS }\end{array}$ & $\begin{array}{l}\text { NS } \\
- \\
-\end{array}$ & 0 & $\begin{array}{l}\text { NS } \\
-\end{array}$ & $\begin{array}{l}\text { NS } \\
-\end{array}$ & $\begin{array}{l}\text { NS } \\
-\end{array}$ \\
\hline $2 \times 1$ & $\begin{array}{l}\mathrm{NS} \\
-\end{array}$ & $\begin{array}{l}\text { NS } \\
\text { - }\end{array}$ & $\begin{array}{l}\text { NS } \\
- \\
-\end{array}$ & $\frac{\mathrm{NS}}{-}$ & 0 & $\frac{\mathrm{NS}}{-}$ & $\frac{\mathrm{NS}}{-}$ \\
\hline $2 \times 3$ & $\begin{array}{l}\text { NS } \\
- \\
-\end{array}$ & NS & $\begin{array}{l}\text { NS } \\
- \\
-\end{array}$ & $\begin{array}{l}\text { NS } \\
-\end{array}$ & $\begin{array}{l}\text { NS } \\
-\end{array}$ & 0 & $\begin{array}{l}\text { NS } \\
--\end{array}$ \\
\hline $3 \times 2$ & $\frac{\text { NS }}{-}$ & $\frac{\text { NS }}{-}$ & $\begin{array}{l}\text { NS } \\
- \\
-\end{array}$ & NS & $\begin{array}{l}\text { NS } \\
- \\
-\end{array}$ & $\frac{\text { NS }}{-}$ & 0 \\
\hline
\end{tabular}

1re ligne: Muscles des lapins mâles;

$2^{\text {e }}$ ligne : Muscles des lapins femelles;

$3^{\mathrm{e}}$ ligne : Muscles des lapins mâles + femelles.

Seuils de signification :

* 5 p. 100 ;

** 1 p. 100 ;

NS (non significatif).

du muscle et la longueur des fibres. Par contre, les individus femelles présentent une corrélation négative entre le nombre de fibres et 1'aire moyenne de section transversale des fibres.

En ce qui concerne le muscle accessorius latissimi dorsi il y a une corrélation positive significative entre le poids du muscle et 1'aire moyenne de section transversale des fibres chez les individus mâles et entre le poids du muscle et la longueur des fibres chez les individus femelles. A nouveau ces derniers présentent une corrélation négative entre le nombre de fibres et l'aire moyenne de section transversale des fibres.

C'est sur ces matrices de corrélation intra que sont menés les calculs de l'analyse factorielle discriminante. 
L'objet de cette analyse est de séparer les 7 groupes de lapins d'après les caractères mesurés sur les divers muscles étudiés et de rechercher l'existence d'une dépendance entre les caractères musculaires étudiés et les 7 groupes de lapins. Pour cela, on calcule de nouvelles variables (vecteurs canoniques), combinaison linéaire des variables initiales.

Au préalable, pour connaître les groupes dont les vecteurs moyens sont significativement différents deux à deux, on utilise le carré de la distance généralisée $\left(\mathrm{D}^{2}\right)$ de MaHal,anobis, qui, à un coefficient multiplicatif près, suit une loi de $\mathrm{F}$ avec $p$ et $\left(n_{1}+n_{2}-p-\mathrm{I}\right)$ degrés de liberté (où $n_{1}$ et $n_{2}$ sont les effectifs de 2 groupes et où $p$ est le nombre de caractères).

Ces groupes sont représentés dans un repère formé par les 2 premiers vecteurs canoniques $F_{1}$ et $F_{2}$. Ce plan a le meilleur pouvoir discriminant chez les individus mâles, femelles et leur ensemble puisqu'il rend compte de plus de $75 \mathrm{p}$. Ioo de la somme des variances intergroupes des variables initiales. En outre, la projection des caractères initiaux est représentée sur ce plan au moyen de leurs coordonnées dans $F_{1}$ et $\mathrm{F}_{2}$. Le caractère $x_{i}(i=\mathrm{I}, 2,3$ et 4$)$ intervient d'autant plus dans la description des nuages de points dans le plan discriminant que la longueur de sa projection, calculée à l'aide de sa corrélation avec les vecteurs canoniques $F_{1}$ et $F_{2}$, est voisine de $I$.

Les résultats des calculs des distances généralisées $\left(\mathrm{D}^{2}\right)$ de MaHaLANoBis et des calculs des vecteurs canoniques sont présentés dans les tableaux 6 et 7 pour les muscles soleus et accessorius latissimi dorsi et dans les tableaux 8 et 9 pour les muscles extensor carpi radialis longus et stylo-hyoideus.

L a représentation graphique des résultats de l'analyse factorielle discriminante pour le muscle soleus est donnée dans la figure I.

On remarque dans le cas du muscle soleus des lapins mâles (fig. I A) que ce sont surtout les caractères $x_{1}, x_{2}$ et, à un moindre degré, le caractère $x_{3}$ qui interviennent dans la description des groupes. Par contre, le caractère $x_{1}$ faiblement corrélé avec $F_{1}$ et $\mathrm{F}_{2} \mathrm{n}^{\prime} \mathrm{y}$ joue pas le rôle. Ainsi, le muscle soleus des lapins mâles Néo-Zélandais se caractérise par un poids élevé et par un plus grand nombre de fibres. A l'opposé, le muscle des individus mâles Communs se caractérise par un poids faible et par un nombre de fibres inférieur. Les individus de ces 2 groupes ont des valeurs sur $x_{3}$ pratiquement identiques.

En ce qui concerne le muscle soleus des lapins femelles (fig. I B) les caractères $x_{2}$ et $x_{4}$ ont la même importance dans la discrimination des groupes. Ainsi, le muscle soleus des individus femelles Néo-Zélandais présente, à nouveau, le poids le plus élevé avec un nombre de fibres important et des fibres de grande longueur. Le muscle soleus des individus Bouscat a un poids moins élevé avec un nombre de fibres plus faible, mais la longueur des fibres reste du même ordre de grandeur que chez les NéoZélandais. Le muscle soleus des individus du groupe $(\mathrm{I} \times 2)$ se distingue de celui des autres groupes surtout par des fibres de plus patite longueur.

En regroupant les données relatives aux muscles soleus des individus mâles et femelles (fig. I C) on observe, que ce sont surtout les caractères $x_{1}$ et $x_{2}$ qui sont importants dans la discrimination des groupes. Le soleus des individus Néo-Zélandais a un poids supérieur à celui des autres groupes et un nombre de fibres plus élevé. Les soleus des individus Bouscat et Commun ont des poids plus faibles et un nombre de fibres inférieur. Les caractères $x_{3}$ et $x_{4}$ sont peu importants dans la description du muscle soleus des groupes, ils sont presque orthogonaux au plan discriminant. 
J. NOUGUỲ, A.-M. ROUS, P. VIGNERON

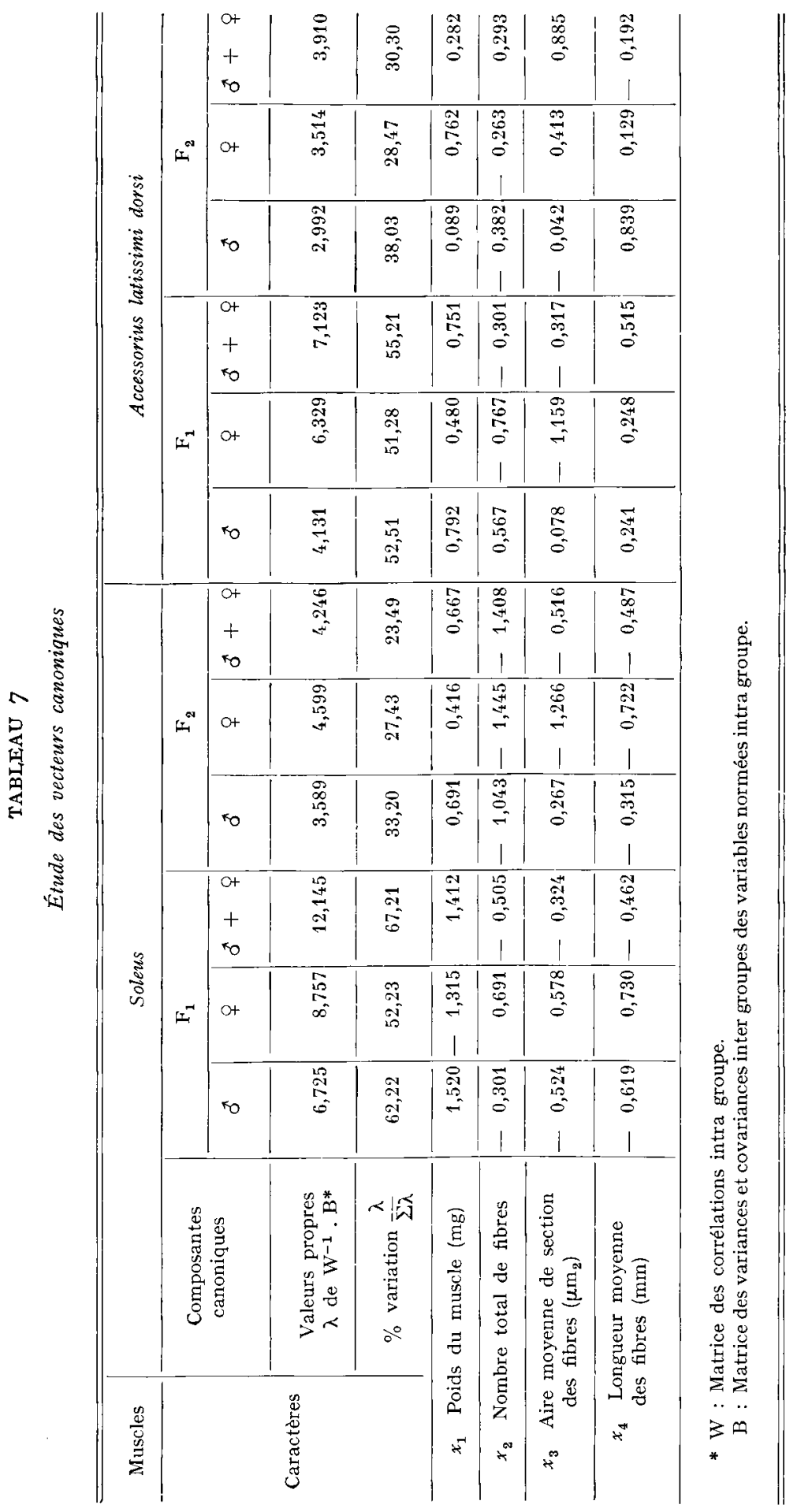


La représentation graphique des résultats de l'analyse factorielle discriminante relative au muscle accessorius latissimi dorsi est donnée dans la figure 2.

Aucune distance séparant les valeurs moyennes des individus des divers groupes, sur les caractères du muscle accessorius latissimi dorsi des lapins mâles, n'est significative et aucun groupe ne se distingue des autres (tabl. 6),
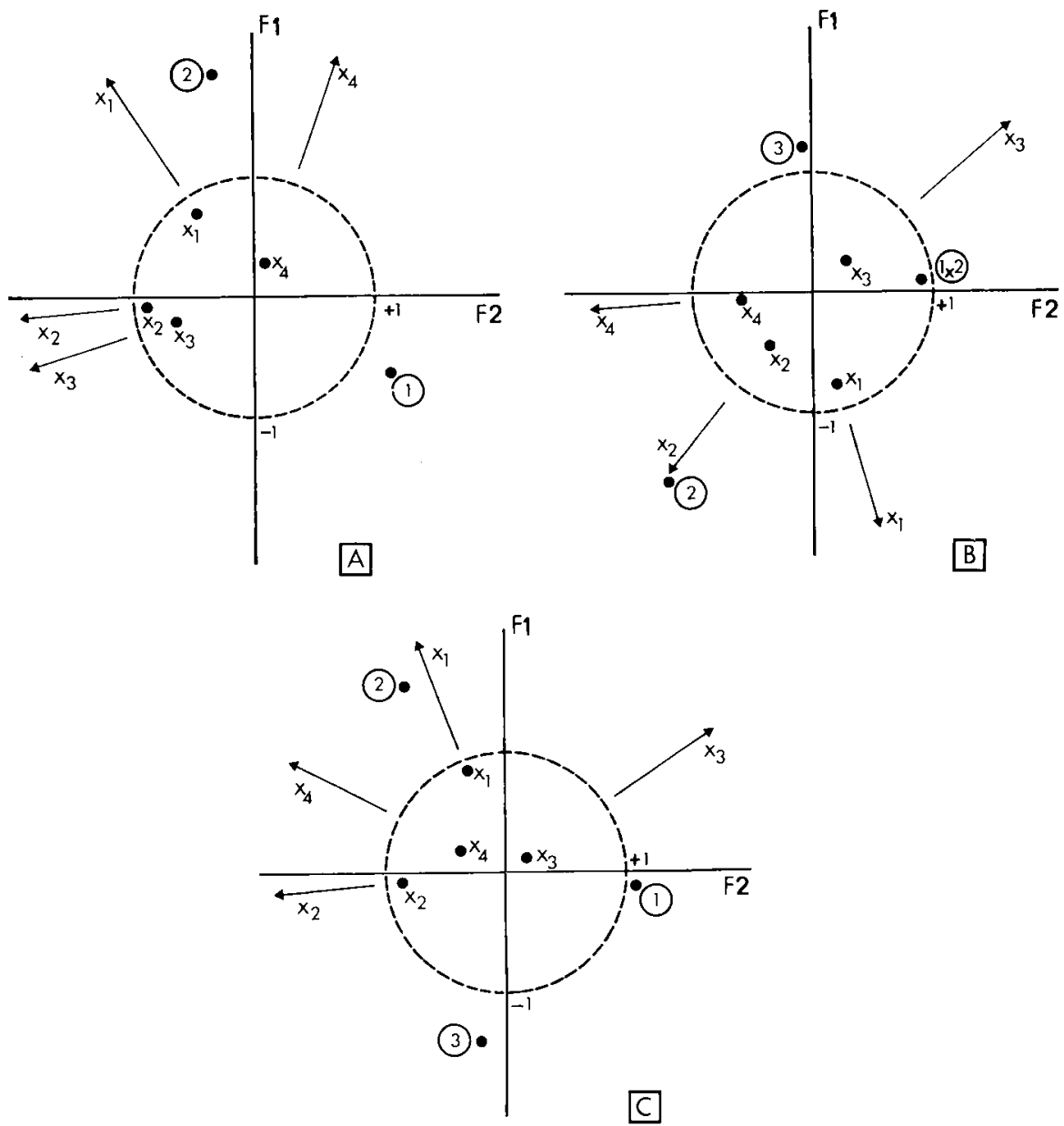

FIG. I. - Représentation des groupes, des projections des axes initiaux

dans le plan des deux premières composantes canoniques et degré d'importance des caractères dans le plan discriminant (cercle de rayon $\mathbf{I}$ )
A : Muscle soleus
B : Muscle soleus
C: Muscle solens of+ $q$.

Par contre, les résultats relatifs au muscle accessorius des lapins femelles (fig. $2 \mathrm{~A}$ ) montrent que, dans l'ordre d'importance, les caractères $x_{3}, x_{1}$ et $x_{4}$ interviennent dans la description du muscle des divers groupes, le caractère $x_{2}$, faiblement corrélé avec $\mathrm{F}_{1}$ et $\mathrm{F}_{2}$, n'y jouant pas de rôle. Le muscle accessorius latissimi dorsi des individus 


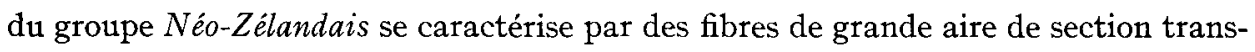
versale et de faible longueur, celui des individus de la race Commune et des croisements $(3 \times 2)$ et $(2 \times$ I $)$ se caractérise par des poids musculaires élevés en relation avec des fibres de grande longueur. Enfin, le muscle accessorius latissimi dorsi, des individus de la race Bouscat et du croisement $(\mathrm{I} \times 2)$ se caractérise par un faible poids en relation avec des valeurs peu élevées de la longueur et de l'aire de section des fibres.
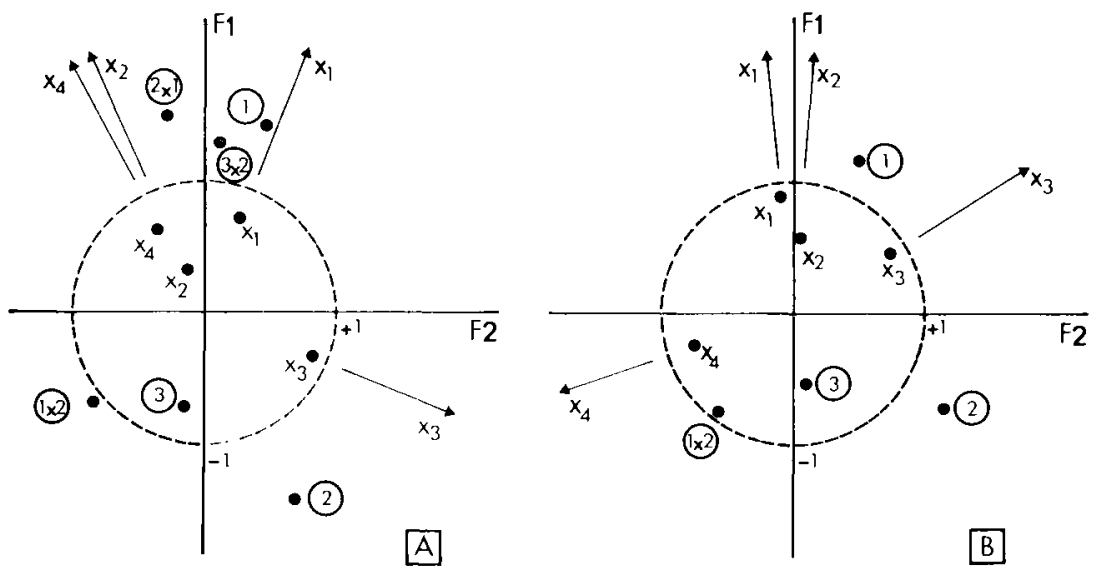

FIG. 2. - Représentation des groupes, des projections des axes initiaux

dans le plan des deux premières composantes canoniques et degrés d'importance des caractères dans le plan discriminant

(cercle de rayon $\mathrm{r}$ )

A : Muscle accessorius latissimi dorsi 우;

$\mathrm{B}$ : Muscle accessorius latissimi dorsi ó + o.

En regroupant les données relatives aux muscles accessorius des individus mâles et femelles (fig. $2 \mathrm{~B}$ ) on constate que ce sont, à nouveau, les caractères $x_{3}, x_{1}$ et $x_{4}$ qui interviennent dans la description du muscle des divers groupes. Les muscles

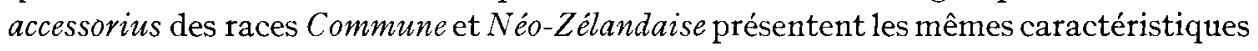
que précédemment. Par contre, il y a, après introduction des données concernant les lapins mâles une rotation du caractère $x_{4}$ vers les groupes Bouscat et $(\mathrm{I} \times 2)$, dont les muscles présentent, en particulier pour ce dernier, un léger accroissement de la longueur des fibres.

En ce qui concerne les muscles extensor carpi radialis longus et stylo-hyoideus, pour lesquels seulement deux caractères ont été étudiés, le poids du muscle et le nombre de fibres, les faits suivants ont été observés :

Chez les lapins mâles les caractères du muscle extensor carpi radialis longus des individus de la race $N e ́ o-Z e ́ l a n d a i s e$ sont différents de ceux des individus de la race Commune par un nombre de fibres supérieur et par un poids plus élevé et de ceux des individus de la race Bouscat par un poids musculaire plus élevé. Le muscle extensor des individus du croisement $(3 \times 2)$ présente comme celui des lapins Néo-Zélandais un nombre de fibres élevé.

Chez les lapins femelles les caractères du muscle extensor carpi radialis longus des individus de la race Bouscat sont différents de ceux des individus des races Néo- 
Zélandaise et Commune par des oppositions surtout entre les poids des muscles et, à un moindre degré, les nombres de fibres ; les individus Bouscat possédant les muscles les plus légers mais avec un nombre de fibres élevé.

\section{TABLEAU 8}

Valeurs de F correspondant aux distances généralisées de Mahalanobis calculées, entre les valeurs moyennes des individus des différents groupes, sur les caractères des muscles extensor carpi radialis longus (au-dessous de la diagonale) et Stylo-hyoideus (au-dessus de la diagonale).

\begin{tabular}{|c|c|c|c|c|c|c|c|}
\hline & 1 & 2 & 3 & $1 \times 2$ & $2 \times 1$ & $2 \times 3$ & $3 \times 2$ \\
\hline 1 & 0 & $\begin{array}{c}5,44^{*} \\
\text { NS } \\
10,41^{* *}\end{array}$ & $\frac{\mathrm{NS}}{-}$ & $\frac{\mathrm{NS}}{-}$ & $\begin{array}{l}\text { NS } \\
-\end{array}$ & $\begin{array}{c}\text { NS } \\
8,02^{*} \\
9,10^{* *}\end{array}$ & $\frac{\text { NS }}{-}$ \\
\hline 2 & $\begin{array}{c}9,55 * * \\
\text { NS } \\
-\end{array}$ & 0 & $\frac{\mathrm{NS}}{4,91 *}$ & $\frac{\text { NS }}{-}$ & $\begin{array}{l}\text { NS } \\
- \\
-\end{array}$ & $\begin{array}{c}\text { NS } \\
\text { NS } \\
7,12^{* *}\end{array}$ & $\begin{array}{l}6,08^{*} \\
\text { NS } \\
\text { NS }\end{array}$ \\
\hline 3 & $\begin{array}{c}\text { NS } \\
10,53^{* *} \\
20,46^{* *}\end{array}$ & $\begin{array}{c}10,92^{* *} \\
7,32^{*} \\
16,64^{* *}\end{array}$ & 0 & $\begin{array}{l}\text { NS } \\
-\end{array}$ & $\frac{N S}{-}$ & $\begin{array}{l}\text { NS } \\
-\end{array}$ & $\begin{array}{l}\text { NS } \\
-\end{array}$ \\
\hline $1 \times 2$ & $\begin{array}{l}\text { NS } \\
-\end{array}$ & $\begin{array}{l}\text { NS } \\
-\end{array}$ & $\begin{array}{l}\text { NS } \\
- \\
-\end{array}$ & 0 & $\begin{array}{l}\text { NS } \\
-\end{array}$ & $\begin{array}{l}\text { NS } \\
- \\
-\end{array}$ & $\begin{array}{l}\text { NS } \\
- \\
-\end{array}$ \\
\hline $2 \times 1$ & $\begin{array}{l}\text { NS } \\
-\end{array}$ & $\begin{array}{l}\text { NS } \\
-\end{array}$ & $\begin{array}{l}\mathrm{NS} \\
- \\
-\end{array}$ & $\begin{array}{l}\text { NS } \\
- \\
-\end{array}$ & 0 & $\begin{array}{l}\text { NS } \\
-\end{array}$ & $\frac{\text { NS }}{-}$ \\
\hline $2 \times 3$ & $\begin{array}{l}\text { NS } \\
-\end{array}$ & $\frac{\mathrm{NS}}{5,83^{*}}$ & $\begin{array}{l}\mathrm{NS} \\
- \\
-\end{array}$ & $\begin{array}{l}\text { NS } \\
- \\
-\end{array}$ & $\begin{array}{l}\text { NS } \\
- \\
-\end{array}$ & 0 & $\begin{array}{l}\text { NS } \\
-\end{array}$ \\
\hline $3 \times 2$ & $\begin{array}{c}8,65^{* *} \\
\text { NS } \\
14,38^{* *}\end{array}$ & $\begin{array}{l}\text { NS } \\
-\end{array}$ & $\frac{\text { NS }}{-}$ & $\frac{\text { NS }}{-}$ & $\begin{array}{l}\text { NS } \\
- \\
-\end{array}$ & $\begin{array}{l}\text { NS } \\
-\end{array}$ & 0 \\
\hline
\end{tabular}

$1^{\text {re }}$ ligne : Muscles des lapins mâles;

$2^{\mathrm{e}}$ ligne: Muscles des lapins femelles;

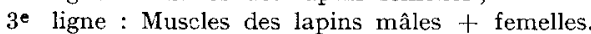

Seuils de signification :

* 5 p. 100 ;

** 1 p. 100 ;

NS (non significatif).

En regroupant les données relatives aux lapins mâles et femelles, on constate à nouveau que le muscle extensor carpi radialis longus des individus de la race Commune a un nombre de fibres inférieur à celui des individus de la race $N e ́ o-Z e ́ l a n d a i s e$ et du produit du croisement de cette dernière avec la race Bouscat. Enfin les lapins 


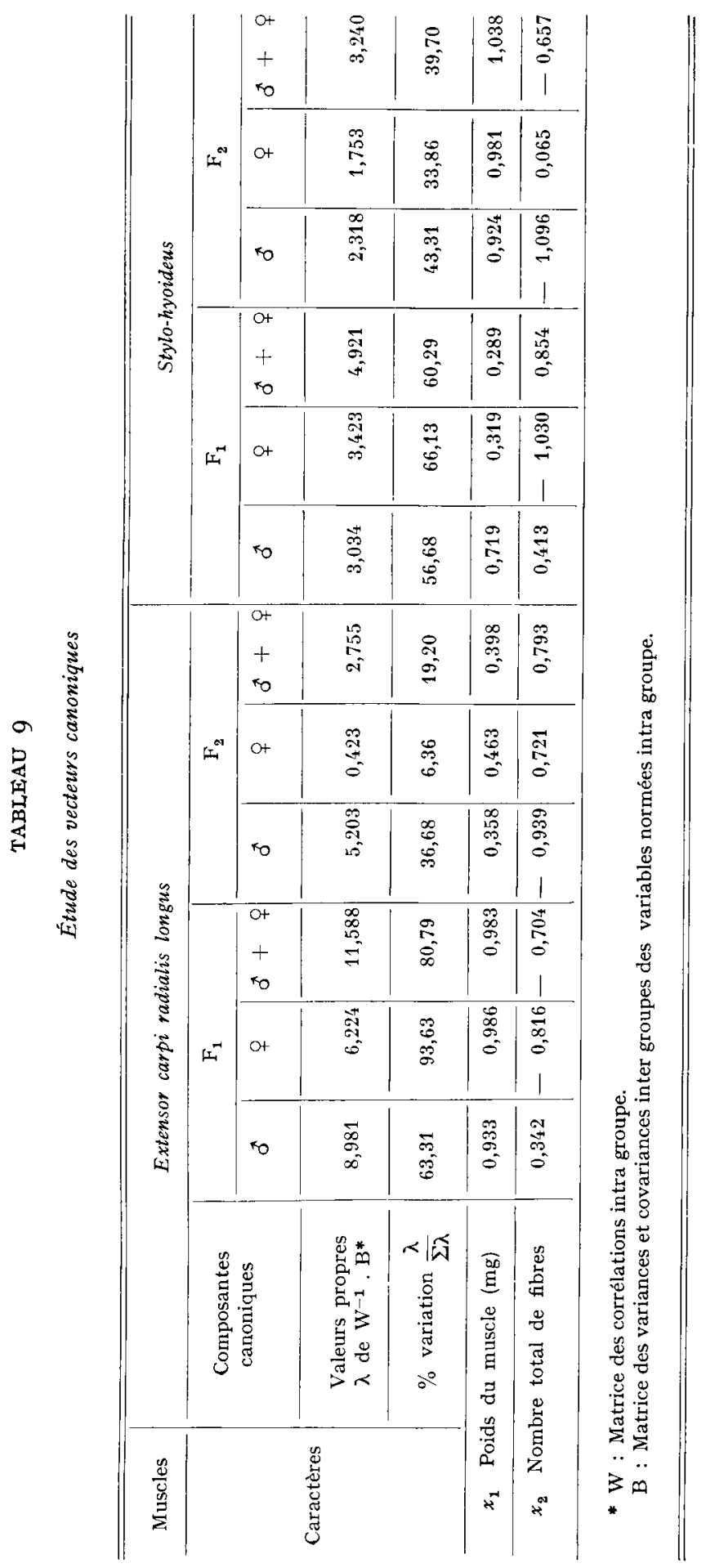


Bouscat possèdent un muscle extensor carpi radialis longus de faible poids mais avec un nombre de fibres élevé. La représentation graphique de ces résultats est donnée dans la figure $3 \mathrm{~A}$.

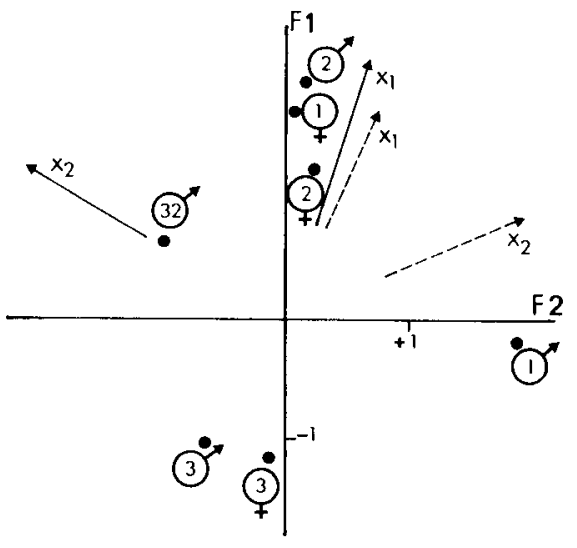

Extensor corpi rodiolis longus

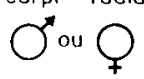

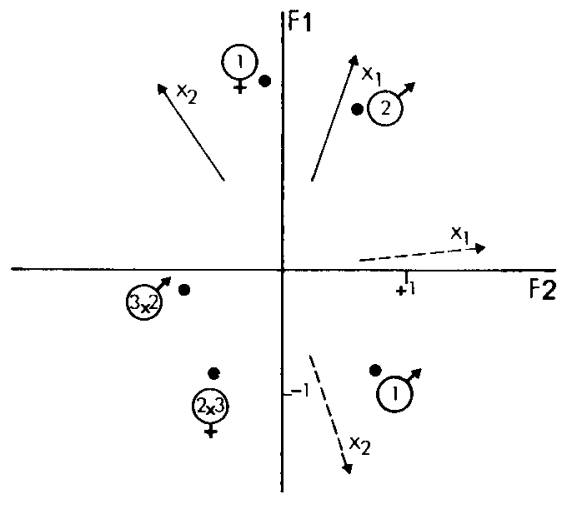

Stylo - hyoideus

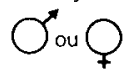

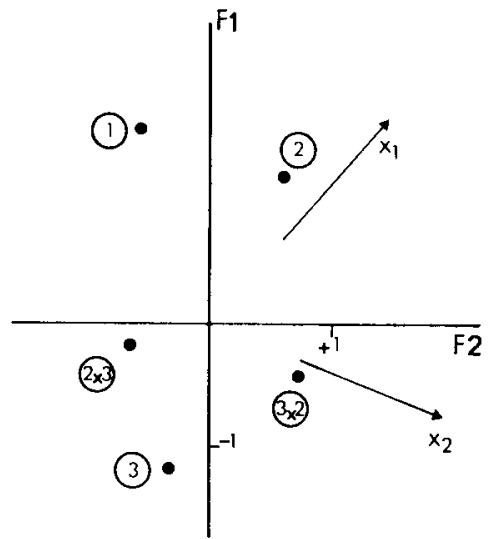

Extensor carpi rodialis longus

ब

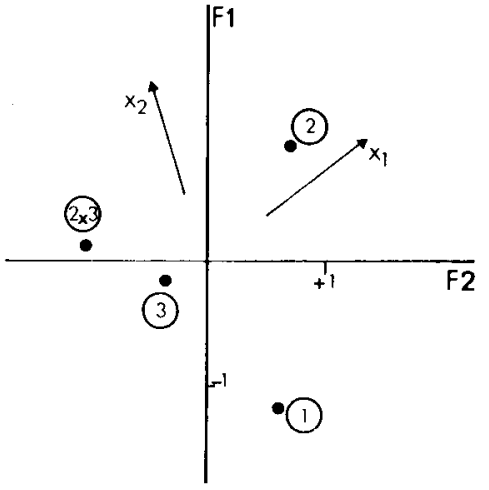

Stylo - hyoideus

B

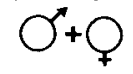

FIG. 3. - Reprêsentation des groupes et des directions des projections des variables dans le plan discriminant $\left(\mathrm{F}_{1}, \mathrm{~F}_{2}\right)$

$$
\longrightarrow \text { Mâles, }
$$

Les caractères des muscles stylo-hyoideus des lapins mâles Néo-Zélandais sont différents de ceux des lapins Commun par un plus grand nombre de fibres et de ceux des individus du croisement $(3 \times 2)$ par un poids supérieur. Chez les lapins femelles les caractères du muscle stylo-hyoideus des individus du croisement $(2 \times 3)$ sont différents de ceux des individus de la race Commune par un plus grand nombre de fibres. Enfin, en regroupant les données relatives aux individus mâles et femelles on observe, 
à nouveau, que les caractères du muscle stylo-hyoideus des Néo-Zélandais sont différents de ceux des Commun et des Bouscat, par un plus grand nombre de fibres. Les lapins du croisement $(2 \times 3)$ ont des muscles stylo-hyoideus différents de ceux des lapins Commun et Néo-Zélandais surtout par le poids du muscle et, à un moindre degré, par le nombre de fibres. La représentation graphique de ces résultats est donnée dans la figure $3 \mathrm{~B}$.

Cette analyse permet d'aboutir aux conclusions générales suivantes : Pour le muscle soleus, c'est le caractère " nombre total de fibres " qui est différent entre les 3 races. Il présente les valeurs les plus élevées chez les lapins Néo-Zélandais et les plus faibles chez les lapins Communs. Le muscle soleus des produits des croisements présente des caractères intermédiaires ou proches de ceux des races, dont ils sont issus.

Par contre, pour le muscle accessorius latissimi dorsi, ce sont les dimensions des fibres qui sont différentes entre les 3 races et certains croisements, mais uniquement chez les lapins femelles. Le muscle des individus Néo-Zélandais se caractérise par des fibres de grande aire de section transversale et celui des individus Communs par un poids plus élevé en liaison avec des fibres de grande longueur. Les muscles des lapins hybrides ne présentent pas de caractères intermédiaires de ceux des parents, dont ils sont issus.

Enfin, les muscles extensor carpi radialis longus et stylo-hyoideus des individus de la race Néo-Zélandaise sont différents de ceux des individus de la race Commune par un plus grand nombre de fibres, sauf en ce qui concerne le muscle extensor carpi radialis longus des lapins femelles. Les muscles des individus Bouscat présentent des caractères intermédiaires entre ceux des Néo-Zélandais et des Communs.

D'autre part, il existe quelques différences, dans les caractères du muscle, liées au sexe. Ainsi, dans le cas du muscle soleus, les individus femelles présentent une variation de la longueur des fibres qui n'existe pas chez les mâles. Le muscle des

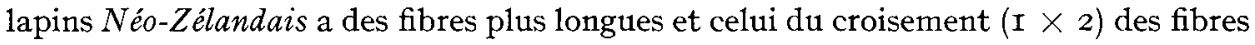
plus courtes que ceux des autres groupes. Seul le muscle accessorius latissimi dorsi des lapins femelles présente des variations, selon la race ou le croisement, des caractères poids du muscle, aire de section transversale et longueur des fibres.

\section{DISCUSSION}

Parmi les races étudiées, les lapins Néo-Zélandais et Commun présentent respectivement des muscles, à l'exception de l'accessorius latissimi dorsi, avec le plus grand nombre de fibres et le plus faible nombre de fibres. On constate ainsi, que la race NéoZélandaise, réputée améliorée pour la production de viande, a des muscles présentant une augmentation du nombre des fibres par rapport aux autres races. Dans le même ordre d'idée, Hanrahan et al. (I973) ont observé dans leur étude de l'influence sur le tissu musculaire, de la sélection de lignées de souris pour un poids corporel élevé, que l'augmentation du poids des muscles des animaux était liée principalement à un accroissement du nombre des fibres musculaires.

D'autre part, LUFF et GoLDSPINK (I970) en étudiant la variation du nombre total de fibres de 4 muscles, prélevés sur des souris adultes de 4 lignées différentes, ont observé qu'à l'intérieur d'une lignée, le nombre de fibres est stable (S. E. de l'ordre 
de 2 p. Ioo de la moyenne) alors qu'entre lignées ce caractère présente des différences importantes et significatives, sauf pour un des muscles étudiés l'extensor digitorum longus. En somme, le nombre total de fibres d'un muscle serait caractéristique d'une lignée. Ces résultats sont partiellement en accord avec ceux obtenus chez le Lapin dans la présente étude. En effet, s'il existe aussi une stabilité assez grande du caractère " nombre de fibres " à l'intérieur d'une race ou d'un croisement, ce caractère n'est pas toujours différent d'une race ou d'un croisement à l'autre.

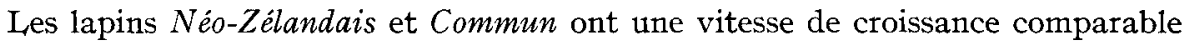
et atteignent, adultes, un poids vif du même ordre de grandeur, alors que les lapins Bouscat, d'un format adulte plus grand, atteignent plus lentement un pourcentage donné de leur poids. Ils présentent donc, au poids d'abattage de $2000 \mathrm{~g}$ un degré de maturité moins prononcé que les autres. Ceci expliquerait les valeurs plus faibles du poids du muscle observées chez les individus Bouscat. Cependant, le nombre de fibres étant définitivement fixé à la naissance ou dans les quelques jours qui suivent, ce caractère n'est pas influencé, au poids d'abattage situé vers 70 jours, par cette différence dans les degrés de maturité, qui peut, par contre, avoir une influence sur les dimensions des fibres.

Pour les divers muscles étudiés, excepté le soleus, il n'y a pas de corrélation significative entre le poids du muscle et le nombre de fibres. L'existence de cette corrélation est très variable. Ainsi (LUFF et GoLDSPINK, I970), dans l'étude des variations du nombre de fibres, chez 4 lignées de souris, ont observé, qu'il n'y avait pas de corrélation positive entre le poids du muscle et le nombre des fibres, alors que, dans un travail antécédent (LUFF et GoLDSPINK, I967) ils avaient constaté en étudiant les mêmes muscles mais chez des souris naines et géantes, qu'il y avait une corrélation positive entre le nombre de fibres et le poids musculaire. A l'opposé (HanRaHan et al., I973) ont observé qu'il n'y avait pas, en général, de corrélation significative entre le nombre de fibres et le poids des muscles anterior tibialis et sternomastoideus de souris appartenant à des lignées sélectionnées pour un poids corporel élevé et faible.

Soulignons également la tendance, chez les individus femelles des différents croisements et races, à une variation en sens inverse du nombre des fibres et de leur section transversale mise en lumière par 1'existence de corrélations négatives significatives. STAUN (I963) a fait une observation similaire pour le muscle longissimus dorsi de porcs de races différentes. Quand le nombre des fibres augmentait dans la section le diamètre des fibres diminuait et vice-versa.

Fin général, il n'existe pas de corrélation significative entre l'aire de section transversale des fibres et leur longueur, sauf pour le muscle accessorius latissimi dorsi des individus femelles de certains croisements et races, où il existe une faible corrélation négative entre ces caractères. Enfin, il n'y a jamais de corrélation significative entre le nombre des fibres et la longueur des fibres.

La longueur des fibres est importante à considérer, puisque les muscles de certains croisements et races présentent uniquement, parmi les dimensions des fibres, une variation de leur longueur sans variation de l'aire de section, comme, par exemple, dans le cas du muscle soleus des lapins femelles.

Enn général, à l'exception du muscle extensor digitorum longus des lapins femelles, il n'y a pas d'influence du sexe sur le nombre des fibres. HanRAHaN et al. (I973) ont fait une observation identique dans le cas des muscles anterior tibialis et sternomastoideus de souris mâles et femelles. Par ailleurs, 1'observation des données individuelles 
permet de remarquer qu'à l'intérieur de chaque race et croisement, il existe des individus qui présentent un caractère " nombre total de fibres » bien supérieur ou inférieur à la moyenne et cette caractéristique se retrouve au niveau des 4 muscles étudiés.

Nos résultats montrent qu'il n'existe pas une règle générale dans la variation des caractères de structure histologique des muscles. Pour certains muscles (soleus) les races et les croisements sont discriminés surtout par le nombre des fibres et pour d'autres muscles (accessorius latissimi dorsi) par les dimensions des fibres. La dissemblance dans la variation des caractères des muscles se retrouve dans les résultats de LUFF et GoLDSPINK (I97o), où, pour les 4 muscles de souris de lignées différentes, seul l'extensor digitorum longus présentait un caractère " nombre de fibres " non différent entre lignées. Par contre, ces mêmes auteurs observaient, dans le cas del'étude des muscles de souris naines et géantes, que c'était le soleus qui ne présentait pas de variation du nombre des fibres.

Rę̧u pour publication en juillet 1973.

\section{REMERCIEMENTS}

Nous remercions M. Millier (Station de Biométrie, Centre national de Recherche forestière) et ses collaborateurs pour les conseils qu'ils ont bien voulu nous apporter.

\section{SUMMARY}

STUDY OF THE VARIATION OF THE NUMBER AND DIMENSIONS

OF MUSCLE FIBERS IN THREE BREEDS OF RABBITS AND THEIR RECIPROCAL CROSSES

The object of the study is to analyze the variation of certain histological characteristics of the soleus, accessorius lattissimi dorsi, extensor carpi radialis longus and stylo-hyoideus muscles in three breeds of rabbits (Common gray, New Zealand, Blanc de Bouscat).

The characters studied are muscle weight, total number of muscle fibers and their average dimensions (area of transveral section and lenght). The first two characters are measured on the 4 muscles; the last 2 are measured only on the soleus and accessorius latissimi dorsi muscles.

An analysis of variance of the data obtained shows, that in the case of the muscles, soleus, extensor carpi radialis longus and stylo-hyoideus the appartenance to a given breed or cross affects the total number of fibers and muscle weight and in the case of the accessorius latissimi dorsi affects the fiber dimension and weight of muscle. Breeds and crosses may then be separated by discriminant factorial analysis in which the variables responsible for this separation are defined.

Results of this analysis for the soleus muscle show that the "total number of fibers " character differs among the three breeds. Its highest values are found in New Z ealand rabbits and its lowest in Common rabbits. In crosses, the soleus muscle presents characters intermediary or similar to those of the breeds of which the crosses are issued.

On the other hand, for the accessorius latissimi dorsi muscle, it is fiber dimension which differs among the three breeds and some crosses, but only in female rabbits. The muscle of New Zealand individuals is characterized by fibers having a large transversal section area and that of Common individuals by a higher weight correlated with longer fibers. Muscles of hybrids do not present the intermediary characteristics of those of the parents of which they are issued.

Finally, except for the extensor carpi radialis longus muscle in female rabbits, the extensor carpi radialis longus and stylo-hyoideus muscles of individuals of the New Zealand breed differ from those of Common individuals by having a greater number of fibers. Muscles of Bouscat rabbits present characters intermediary between those of New Zealand and Common. The results of this study show that the histological characters of a muscle, especially the number of fibers, may present variations of genetic origin among breeds of rabbits. 


\section{RÉFÉRENCES BIBLIOGRAPHIQUES}

DorozynskA D., LiszkA E., I97o. Measurements of muscle fibre transverse sections in rabbits of both early and late maturity and their crossbreeds. Acta agr. silvest. Ser. zootech., Krakow, 10, 73-82.

Hammond J., Appleton A. B., 1932. Growth and development of mutton qualities in the sheep. Oliver and Boyd, Edinbourg, 595 p.

Hanrahan J. P., Hooper A. C., Mc Carthy J. C., 1973. Effects of divergent selection for body weight on fibre number and diameter in two mouse muscles. Anim. Prod., 16, 7-16.

Joubert D. M., 1956. An analysis of factors influencing postnatal growth and development of the muscle fibre. J.agric. Sci. Camb., 47, 59-102.

Luff A. R., Goldspink G., I967. Large and small muscles. Life Sci., 6, г821-1826.

LUfF A. R., Goldspink G., 1970. Total number of fibres in muscles of several strains of mice. $J$. Anim. Sci., 30, 89I-893.

Mauch A., Marinesco J., 1934. Ann. Inst. nat. Zootech., Roumain, 3, 154, in : Anim. Breed. Abstr., 19 , I.

Mizuno T., Hrкami Y., ig67. Growth of muscle fibre in chik embryos. Jap. J. Zootech. Sci., 38, 553558.

Nougutss J., 1972. Étude de l'évolution du nombre des fibres musculaires au cours de la croissance postnatale du muscle chez le Lapin. C.R. Soc. Biol., 166, 165-172.

Nouguès J., I973. Étude histologique de la croissance postnatale des muscles soleus et accessorius latissimi dorsi chez le Lapin Commun. Ann. Biol. anim. Bioch. Biophys., 13, 37-50.

Sмiтн J. H., 1963. Relation of body size to muscle cell size and number in the chicken. Poult. Sci, 42, 282-290.

Staun H., rg63. Various factors affecting number and size of muscle fibres in the pig. Acta. Agric. Scand., 13, 293-322. 